\title{
Performance Analysis of Iterative Turbo Decoding Stopping Criteria with Quadrature Amplitude Modulation
}

\author{
Mohamad Yusuf Mat Nasir, Roslina Mohamad, Murizah Kassim, Nooritawati Mat Tahir
}

\begin{abstract}
Iterative turbo decoding stopping criteria are known to be used in early termination of the turbo decoding iteration without sacrificing its performance. However, most of these stopping criteria were tested using low modulation techniques. In this paper, several stopping criteria were tested using higher modulation techniques, such as quadrature amplitude modulation (QAM). The sign-change-ratio (SCR), hard-decision-aided (HDA), and cross-entropy (CE) stopping criteria were simulated with 4-QAM, 8-QAM, and 16-QAM in additive white Gaussian noise. From the simulation results, HDA and SCR are capable of terminating early at various stages of noise while maintaining the bit error rate performance. However, CE fails to terminate early in high-noise channels and only terminates early when the output of the turbo decoder is in a lownoise channel.
\end{abstract}

Index Terms - Iterative decoding, turbo codes, quadrature amplitude modulation, stopping criterion

\section{INTRODUCTION}

Back in 1993, a paper entitled "Near Shannon Limit Errorcorrecting Coding and Decoding: Turbo-codes" was published. It introduced turbo codes to the world[1]. This introduction brought a major improvement to communication systems [2], such as excellent bit error rate (BER) performance because of the iterative decoding, concatenated coding, and maximum a posteriori (MAP) decoders [1]. However, these improvements required much delay and computation complexity for decoding process [3]. These major problems were further improved by many researchers by adding a stopping criterion to the decoder [4]-[7], such as sign-change-ratio (SCR), hard-decision-aided (HDA), and cross-entropy (CE) [4], [6]. Most of the research on the iterative turbo decoding stopping criteria is limited to testing in low modulation techniques, such as binary phase-shift keying (BPSK) [1]-[3], [6], [7]. For example, research in [4] tested CE, HDA and SCR stopping criteria using BPSK modulation. Another research [6] also tested the enhancement

This manuscript is submitted on $14^{\text {th }}$ August 2019 and accepted on $04^{\text {th }}$ December 2019. This work was supported in part by the Ministry of Higher Education (MOHE) for supporting this research through FRGS grant number FRGS/1/2017/ICT02/UITM/02/2.

Mohamad Yusuf Mat Nasir, Roslina Mohamad, Murizah Kassim, and Nooritawati Mat Tahir are with the Faculty of Electrical Engineering, Universiti Teknologi MARA, 40450 Shah Alam, Malaysia (e-mail: noori422@uitm.edu.my). of CE stopping using the BPSK modulation.

The researcher in [8] presented the performance analysis on the sign difference ratio (SDR) stopping criterion in three different methods of Max-Log MAP algorithm where each method had different numbers of mathematical operations. Method 1 required seven mathematical operations, method 2 required three mathematical operations, and method 3 used two mathematical operations. These three methods are then tested using three different modulations: BPSK, quadrature phase-shift keying (QPSK), and 16- quadrature amplitude modulation (QAM). The research in [9] discussed the performance analysis of HDA stopping in BPSK modulation by using half iteration. A robustness test and performance analysis of the enhancement of CE stopping criterion were done in [6]. Research in [10] analysed the performance analysis of SDR in additive white Gaussian noise (AWGN).

L. H. Abderramane tested CE stopping criterion in AWGN and Rayleigh channels [11]. The turbo codes used two sets of interleaver: the $\mathrm{CE}$ interleaver and the Dithered golden interleaver. Yuejun Wei et al. [12] applied HDA, cyclic redundancy check (CRC), and parity-check stopping (PCS) with Max-log-MAP and log-MAP decoding. The researcher in [13] used a CE minimisation method for turbo decoding.

Most of the research on the iterative turbo decoding stopping criteria are limited to testing in low modulation techniques, such as BPSK [8]. Hence, the lack of information about the performance of the stopping criteria in the higher modulation has led to the purpose of this paper. The rest of this paper is organised as follows. In Section II, the simulation parameters used in the research are elaborated. In Section III, the results of the CE, SCR and HDA stopping criteria with 4, 8 and 16-QAM are analysed and compared. Finally, the conclusion is drawn in Section IV.

\section{SimUlation PARAMETERS}

Table I shows all the parameters used in the simulation. The research chose the well-known stopping criteria such as $\mathrm{CE}$, SCR and HDA. The stopping criteria algorithms for CE, SCR and HDA can be referred in [4]. The thresholds for CE and SCR were set to 0.0001 and 0.012 , respectively. One million random binary data with three different modulations (4-QAM, 8-QAM, and 16-QAM) were applied in the simulation. Meanwhile, for the frame size, 1000, 2000 and 10000 were used for each data transmission. The turbo code generator and 
code rate were $[31,17]$ and $1 / 3$, respectively. The codes were simulated under the AWGN channel and the maximum iteration number, $I_{\max }$ was set to eight.

TABLE I

SIMULATION PARAMETERS

\begin{tabular}{cc}
\hline \hline Parameters & Name/ Value \\
\hline Stopping Criteria & CE, SCR, HDA \\
Modulation & 4-QAM, 8-QAM, 16-QAM \\
Frame size & $1000(1 \mathrm{~K}), 10000(10 \mathrm{~K})$ \\
Code Generator & {$[31,17]$} \\
Code rate & $1 / 3$ \\
Channel model & AWGN \\
Maximum iteration number & 8 \\
\hline \hline
\end{tabular}

\section{RESULTS AND DISCUSSION}

In this section, the performance of turbo codes with three different stopping criteria, modulations and frame sizes are compared. The performance of each stopping criterion is analysed based on the BER and average iteration number (AIN). The formula for saving iteration percentage $(\alpha)$ is derived in (1).

$$
\alpha=\frac{\left|I_{n}-\mathrm{I}_{\max }\right|}{I_{\max }} \times 100
$$

Where $I_{n}$ is the AIN at $n$ of the energy per bit to noise power spectral density ratio (Eb/No) and $n$ is the Eb/No level.

\section{A. Performance of CE Stopping Criterion}

The AIN of turbo codes with CE stopping criterion for 4QAM, 8-QAM, and 16-QAM modulations are shown in Figures 1, 2, and 3, respectively. Meanwhile, the BER performance of turbo codes with CE stopping criterion for 4QAM, 8-QAM and 16-QAM modulations are shown in Figures 4, 5 and 6, respectively. From Fig. 1, the AIN for $1 \mathrm{~K}$ frame size and 4-QAM start to reduce at $-0.2 \mathrm{~dB}$ and for $10 \mathrm{k}$ frame size at $0 \mathrm{~dB}$. At $3 \mathrm{~dB} \mathrm{~Eb} / \mathrm{No}$, both $1 \mathrm{~K}$ and $10 \mathrm{~K}$ can terminate early at the second iteration, which can save $72 \%$ AIN from the fixed stopping criterion.

Meanwhile, for Fig. 2, it can be observed that the iteration number for 8 -QAM with $1 \mathrm{~K}$ frame size starts to reduce at 1 $\mathrm{dB}$ and for $10 \mathrm{~K}$ frame size at $1.2 \mathrm{~dB}$. At $4 \mathrm{~dB} \mathrm{~Eb} / \mathrm{No}$, both $1 \mathrm{~K}$ and $10 \mathrm{~K}$ can terminate early at 2.1 and 2.3 iteration, respectively, which can save around $71-74 \%$ from a fixed stopping criterion. From Fig. 3, the AIN performance for frame 1K, 16-QAM turbo code with $\mathrm{CE}$ stopping starts to reduce at $1.2 \mathrm{~dB}$, meanwhile, for a $10 \mathrm{~K}$ frame, it starts at $2 \mathrm{~dB}$. At $4 \mathrm{~dB} \mathrm{~Eb} / \mathrm{No}$, both $1 \mathrm{~K}$ and $10 \mathrm{~K}$ can terminate early at 2.4 and 3 iteration, respectively, which can save around $63-70 \%$ from a fixed stopping criterion.

For BER performances of 4-QAM, as shown in Fig. 4, it can be observed that the BER for CE and fixed stopping for $1 \mathrm{~K}$ frame size are the same. For $10 \mathrm{~K}$ frame size, there is a slight difference in the BER start at $0 \mathrm{~dB}$. For 8-QAM, the BER performance for $\mathrm{CE}$ and fixed stopping criteria for $1 \mathrm{~K}$ and $10 \mathrm{~K}$ frame sizes are almost the same as depicted in Fig. 5.
The same performance is also achieved by 16-QAM turbo codes, as shown in Fig. 6.

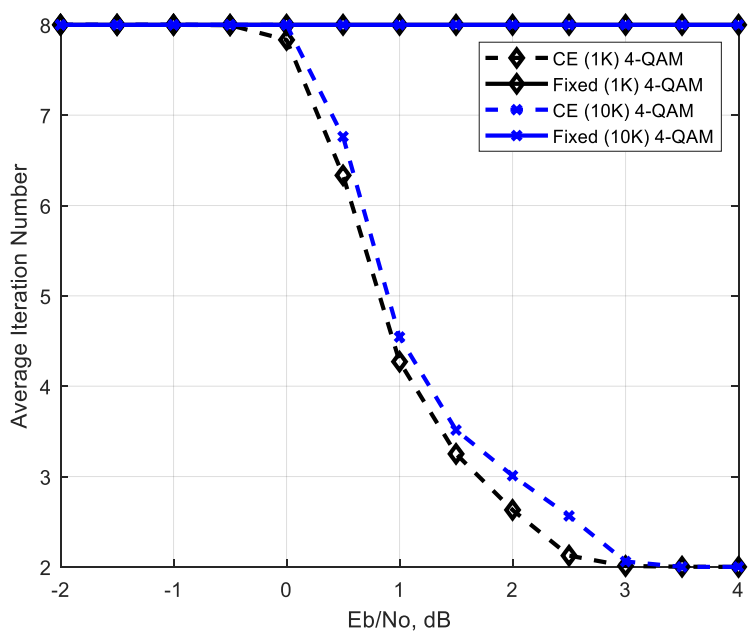

Fig. 1. AIN performance for 4-QAM turbo codes with $\mathrm{CE}$ and fixed stopping criteria.

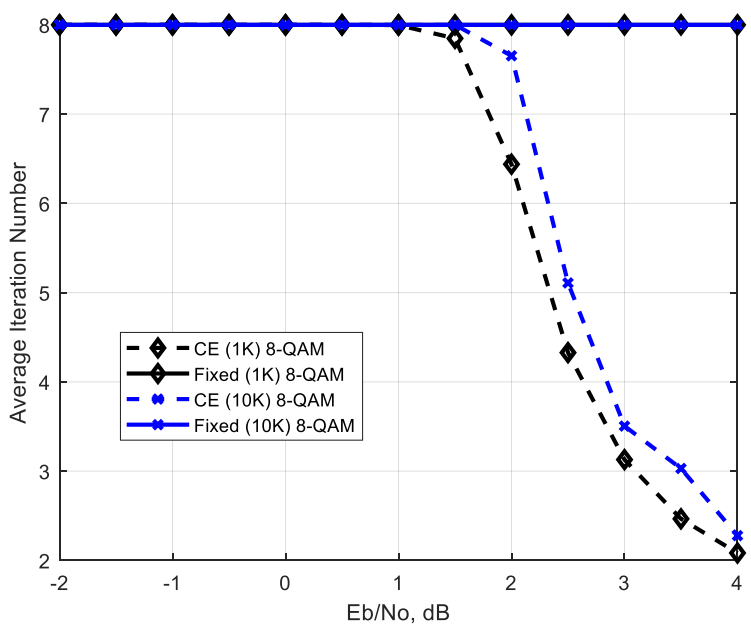

Fig. 2. AIN performance for 8-QAM turbo codes with $\mathrm{CE}$ and fixed stopping criteria.

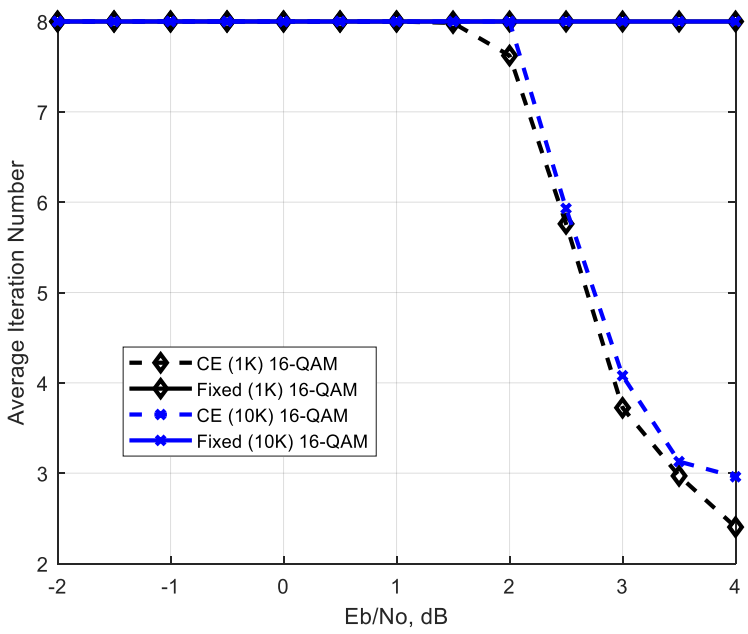

Fig. 3. AIN performance for 16-QAM turbo codes with $\mathrm{CE}$ and fixed stopping criteria. 


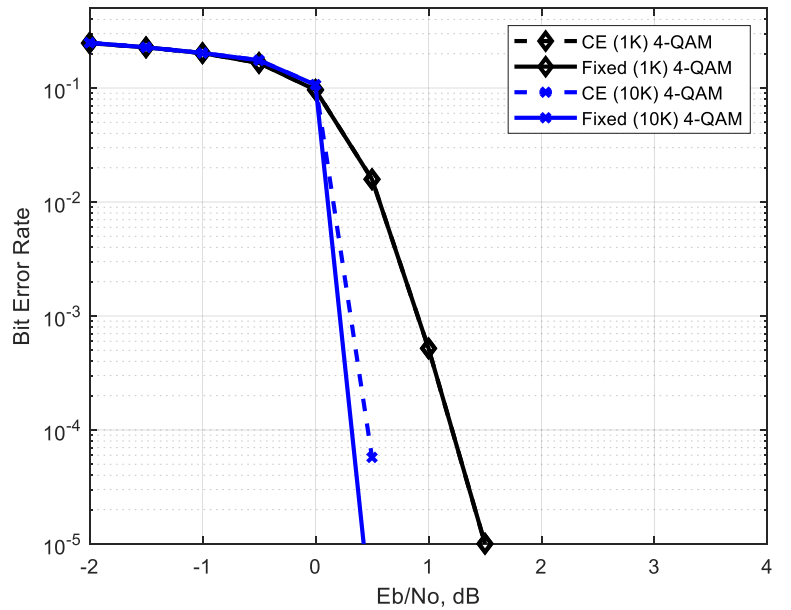

Fig. 4. BER performance for 4-QAM turbo codes with CE and fixed stopping criteria.

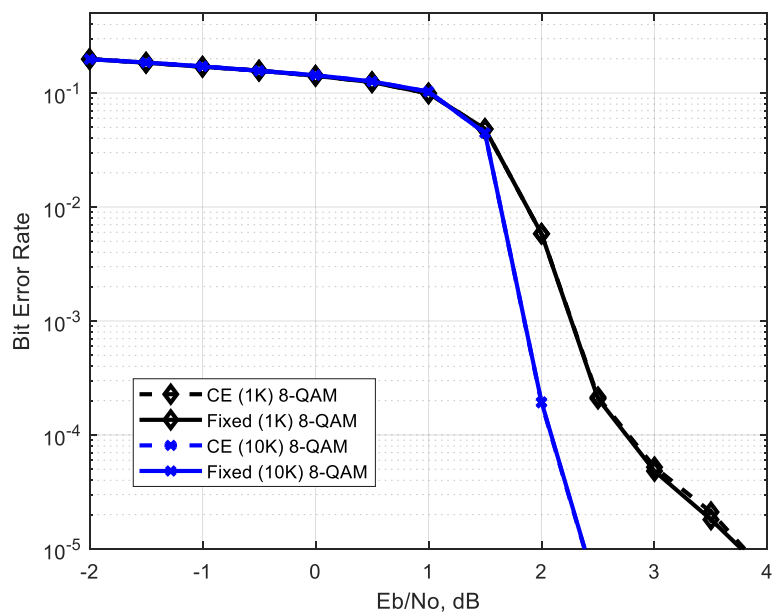

Fig. 5. BER performance for 8-QAM turbo codes with CE and fixed stopping criteria.

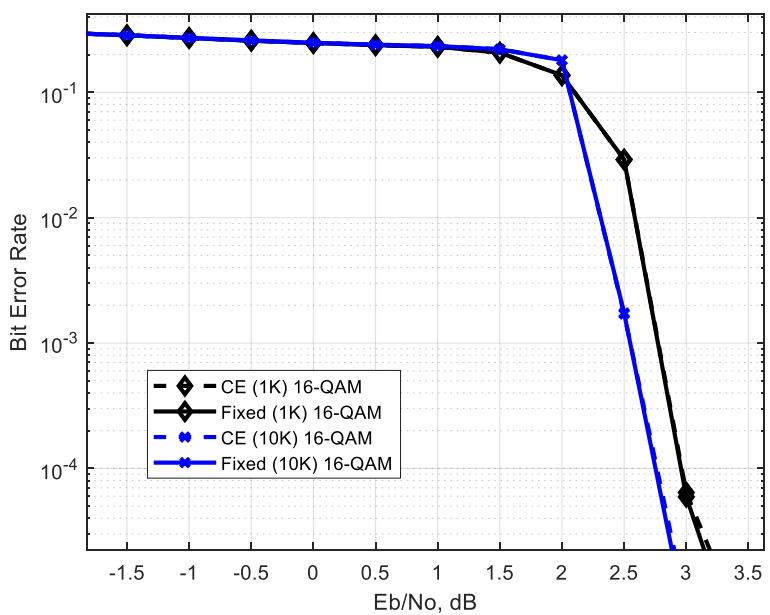

Fig. 6. BER performance for 16-QAM turbo codes with CE and fixed stopping criteria.

\section{B. Performance of SCR Stopping Criterion}

The AIN of turbo codes with SCR stopping criterion for 4QAM, 8-QAM and 16-QAM modulations are shown in Figures 7,8 and 9, respectively. Meanwhile, the BER performance of turbo codes with CE stopping criterion for 4QAM, 8-QAM and 16-QAM modulations are shown in Figures 10, 11 and 12, respectively. From Fig. 7, the SCR for 4-QAM with $1 \mathrm{~K}$ and $10 \mathrm{~K}$ frame sizes is capable of terminating early at $3 \mathrm{AIN},-2 \mathrm{~dB}$. The AIN for both frame sizes starts to increase and achieves maximum AIN at $-1.2 \mathrm{~dB}$. It can be observed that the iteration number for $1 \mathrm{~K}$ frame size starts to reduce at $-0.2 \mathrm{~dB}$. Meanwhile, for $10 \mathrm{~K}$ frame size, it starts to reduce at $0 \mathrm{~dB}$. At $4 \mathrm{~dB} \mathrm{~Eb} / \mathrm{No}$, both $1 \mathrm{~K}$ and $10 \mathrm{~K}$ can terminate early at the second iteration, which can save around $72 \%$ from the fixed stopping criterion.

Meanwhile, for Fig. 8, the SCR for 8-QAM with $1 \mathrm{~K}$ and $10 \mathrm{~K}$ frame sizes is capable of terminating early at $3 \mathrm{AIN}$, $2 \mathrm{~dB}$. The AIN for both frame sizes starts to increase and achieves maximum AIN at $0.2 \mathrm{~dB}$. It can be observed that the iteration number for $1 \mathrm{~K}$ frame size starts to reduce at $1 \mathrm{~dB}$. Meanwhile, for a $10 \mathrm{~K}$ frame size, it starts to reduce at $1.2 \mathrm{~dB}$. At $4 \mathrm{~dB} \mathrm{~Eb} / \mathrm{No}$, both $1 \mathrm{~K}$ and $10 \mathrm{~K}$ can terminate early at the second iteration, which can save around $72 \%$ from the fixed stopping criterion. From Fig. 9, the SCR for 16-QAM with 1K and $10 \mathrm{~K}$ frame sizes is capable of terminating early at $3 \mathrm{AIN}$, $2 \mathrm{~dB}$. The AIN for both frame sizes starts to increase and achieves maximum AIN at $0.2 \mathrm{~dB}$. It can be observed that the iteration number for $1 \mathrm{~K}$ frame size starts to reduce at $1.2 \mathrm{~dB}$, meanwhile, for a $10 \mathrm{~K}$ frame size, it starts to reduce at $2 \mathrm{~dB}$. At $4 \mathrm{~dB} \mathrm{~Eb} / \mathrm{No}$, both $1 \mathrm{~K}$ and $10 \mathrm{~K}$ can terminate early at the third iteration, which can save around $63 \%$ from the fixed stopping criterion.

For BER performances of 4-QAM as shown in Fig. 10, it can be observed that the BER for SCR and fixed stopping for $1 \mathrm{~K}$ frame size are the same. For $10 \mathrm{~K}$ frame size, there is a slight difference in BER starting at $0 \mathrm{~dB}$ and increasing until $0.6 \mathrm{~dB}$ at $\mathrm{BER}=10^{-2}$. For 8-QAM, the BER performances for $\mathrm{SCR}$ and the fixed stopping criteria for $1 \mathrm{~K}$ have a slight difference. For the $10 \mathrm{~K}$ frame size, there is a slight difference in the BER starting at $1.2 \mathrm{~dB}$, increasing until $0.2 \mathrm{~dB}$ at BER $=$ $10^{-2}$, as depicted in Fig. 11. The same performance is also achieved by 16-QAM turbo codes. Meanwhile, it can be observed that the BER for SCR and fixed stopping for $10 \mathrm{~K}$ frame size are almost the same. For $1 \mathrm{~K}$ frame size, there is a slight difference in BER starting at $2.2 \mathrm{~dB}$ and increasing until $0.3 \mathrm{~dB}$ at $\mathrm{BER}=10^{-2}$, as shown in Fig. 12 . 


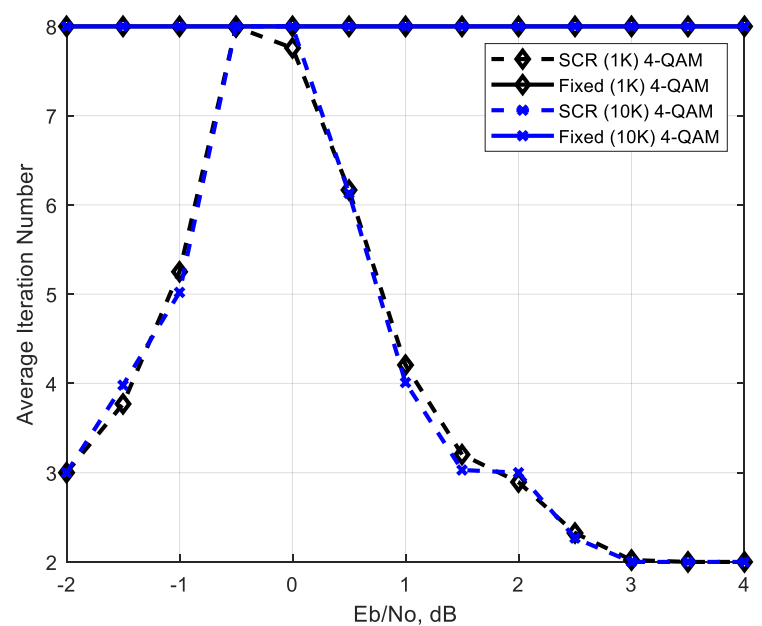

Fig. 7. AIN performance for 4-QAM turbo codes with SCR and fixed stopping criteria.

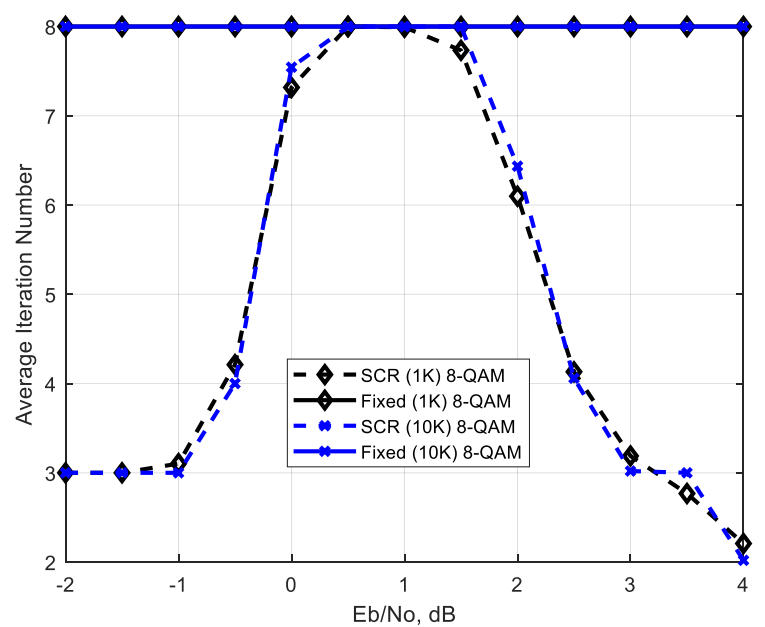

Fig. 8. AIN performance for 8-QAM turbo codes with SCR and fixed stopping criteria.

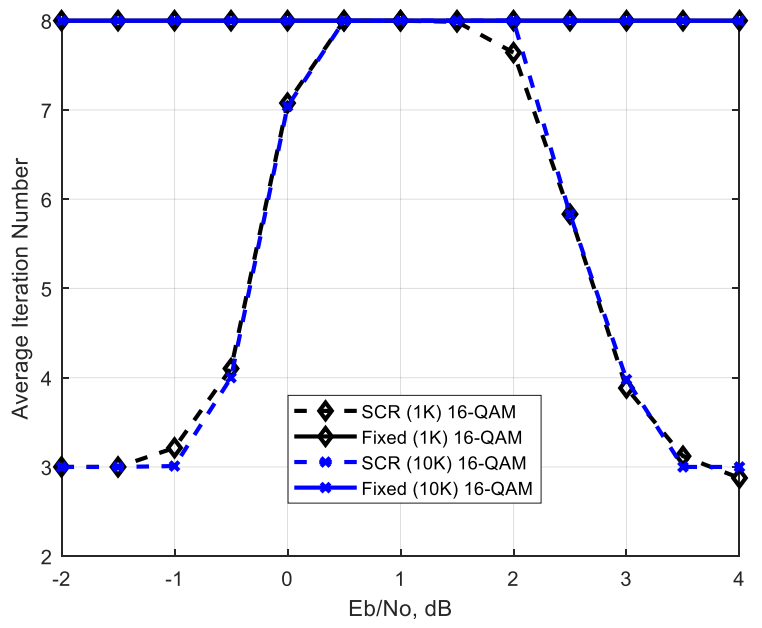

Fig. 9. AIN performance for 16-QAM turbo codes with SCR and fixed stopping criteria.

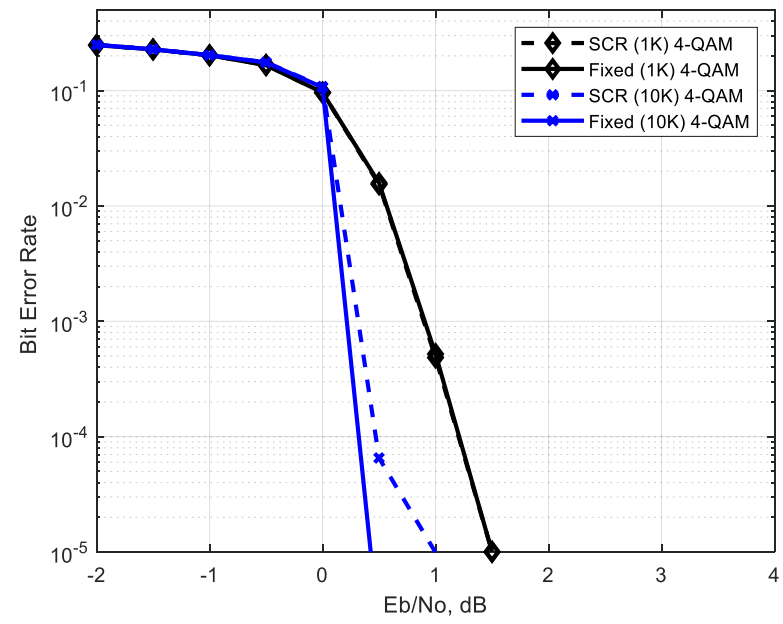

Fig. 10. BER performance for 4-QAM turbo codes with SCR and fixed stopping criteria.

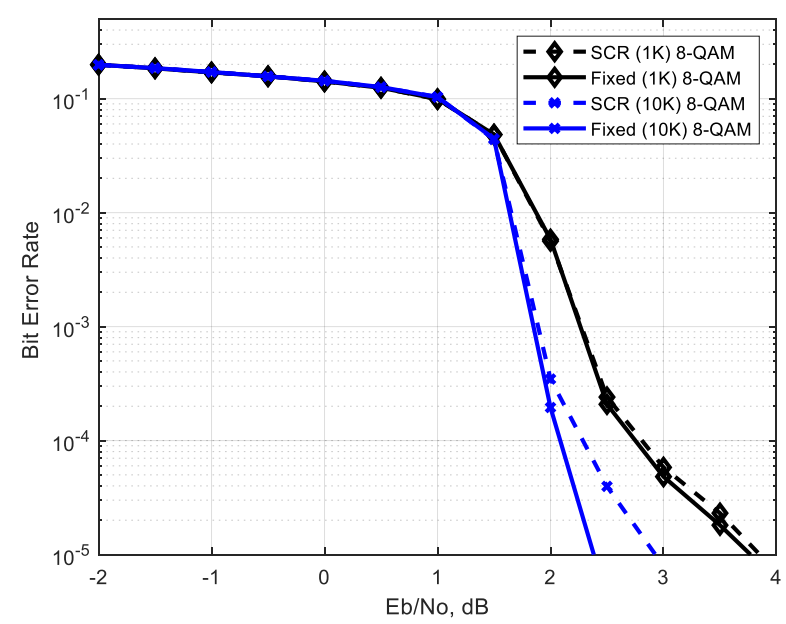

Fig. 11. BER performance for 8-QAM turbo codes with SCR and fixed stopping criteria.

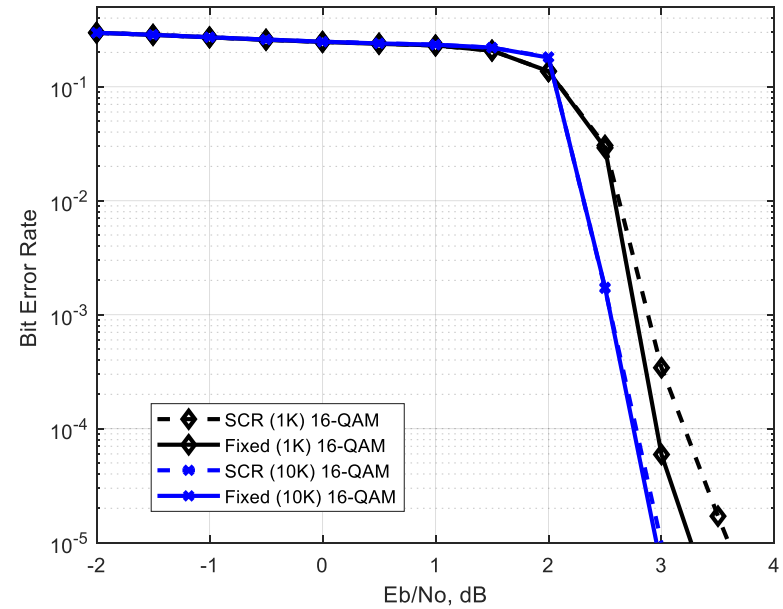

Fig. 12. BER performance for 16-QAM turbo codes with SCR and fixed stopping criteria. 


\section{Performance of HDA Stopping Criterion}

The AIN performances of turbo codes with HDA stopping criterion for 4-QAM, 8-QAM, and 16-QAM modulations are shown in Figures 13, 14, and 15, respectively. The BER performances of turbo codes with HDA stopping criterion for 4-QAM, 8-QAM, and 16-QAM modulations are shown in Figures 16, 17 and 18, respectively. From Fig. 13, at $-2 \mathrm{~dB}$, the HDA for 4-QAM with $1 \mathrm{~K}$ and $10 \mathrm{~K}$ frame sizes is capable of terminating early at 3.3 and 4 AIN, respectively. The AIN for $1 \mathrm{~K}$ and $10 \mathrm{~K}$ starts to increase and achieves maximum AIN at $-0.2 \mathrm{~dB}$ and $-1 \mathrm{~dB}$, respectively. It can be observed that the iteration number for $1 \mathrm{~K}$ frame size starts to reduce at $-0.2 \mathrm{~dB}$ while for a $10 \mathrm{~K}$ frame size it starts to reduce at $0 \mathrm{~dB}$. At $4 \mathrm{~dB}$, both $1 \mathrm{~K}$ and $10 \mathrm{~K}$ can terminate early at the second iteration, which can save around $72 \%$ from the fixed stopping criterion.

Meanwhile for Fig. 14, at $2 \mathrm{~dB}$, the HDA for 8-QAM with $1 \mathrm{~K}$ and $10 \mathrm{~K}$ frame sizes is capable of terminating early at 3 and 3.2 AIN, respectively. The AIN for both frame sizes starts to increase and achieves a maximum AIN at $0 \mathrm{~dB}$. It can be observed that the iteration number for $1 \mathrm{~K}$ frame size starts to reduce at $1 \mathrm{~dB}$ while for the $10 \mathrm{~K}$ frame size it starts to reduce at $1.2 \mathrm{~dB}$. At $4 \mathrm{~dB} \mathrm{~Eb} / \mathrm{No}$, both $1 \mathrm{~K}$ and $10 \mathrm{~K}$ can terminate early at 2.2 and 3 AIN, which can save around $69 \%$ and $63 \%$ from the fixed stopping criterion, respectively. From Fig. 15, at $2 \mathrm{~dB}$, the HDA for 16-QAM with $1 \mathrm{~K}$ and $10 \mathrm{~K}$ frame sizes is capable of terminating early at 2.8 and $3.2 \mathrm{AIN}$, respectively. The AIN for both frame sizes start to increase and achieves maximum $\mathrm{AIN}$ at $0 \mathrm{~dB}$. It can be observed that the iteration number for $1 \mathrm{~K}$ frame size starts to reduce at 1.2 $\mathrm{dB}$ while for the $10 \mathrm{~K}$ frame size it starts to reduce at $2 \mathrm{~dB}$. At $4 \mathrm{~dB} \mathrm{~Eb} / \mathrm{No}$, both $1 \mathrm{~K}$ and $10 \mathrm{~K}$ can terminate early at 3 and 3.1 AIN, which can save around $63 \%$ and $61 \%$ from the fixed stopping criterion, respectively.

For BER performance of 4-QAM, as shown in Fig. 16, it can be observed that the BER for HDA and fixed stopping for $1 \mathrm{~K}$ frame size are the same. For $10 \mathrm{~K}$ frame size, there is small BER degradation starting at $\mathrm{BER}=10^{-1}$ and increasing until $0.2 \mathrm{~dB}$ at $\mathrm{BER}=10^{-4}$. For $8-\mathrm{QAM}$, the BER performance for HDA and the fixed stopping criteria for $1 \mathrm{~K}$ and $10 \mathrm{~K}$ have similar performance as depicted in Fig. 17. The same performance is also achieved by 16-QAM turbo codes. Meanwhile, it can be observed that for the BER for SCR and fixed stopping for $1 \mathrm{~K}$ frame size, there is a slight difference in BER starting at 3.4, as depicted in Fig. 18.

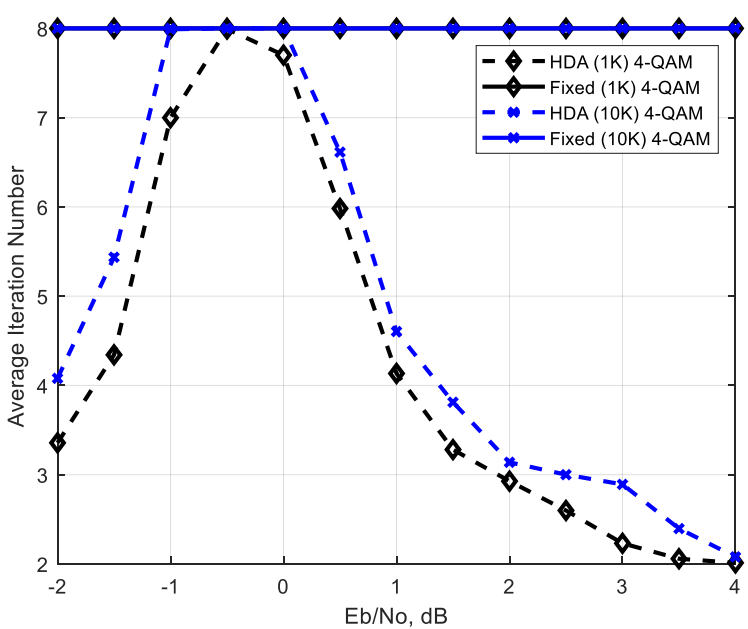

Fig. 13. AIN performance for 4-QAM turbo codes with HDA and fixed stopping criteria.

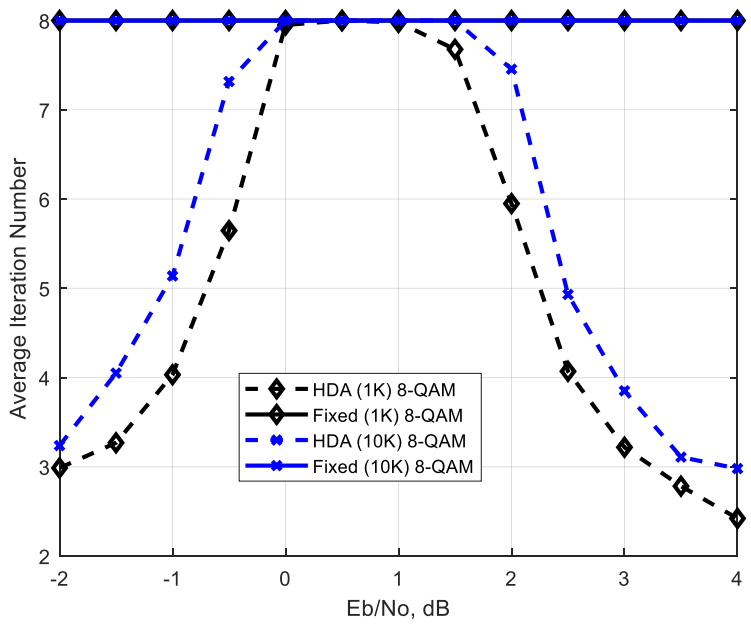

Fig. 14. AIN performance for 8-QAM turbo codes with HDA and fixed stopping criteria.

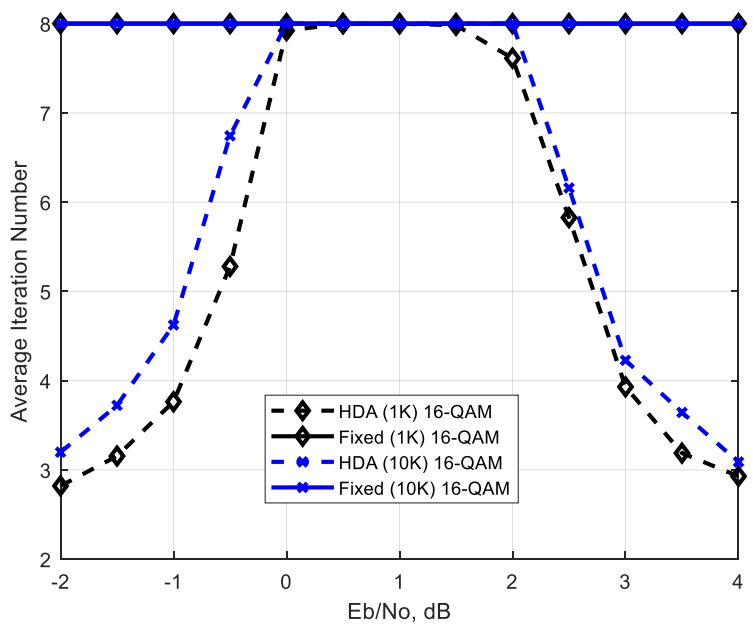

Fig. 15. AIN performance for 16-QAM turbo codes with HDA and fixed stopping criteria. 


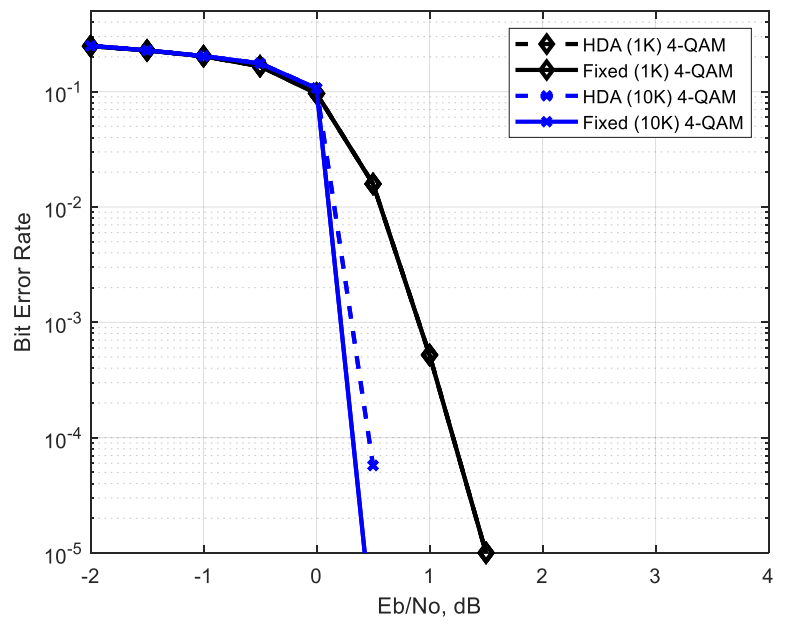

Fig. 16. BER performance for 4-QAM turbo codes with HDA and fixed stopping criteria.

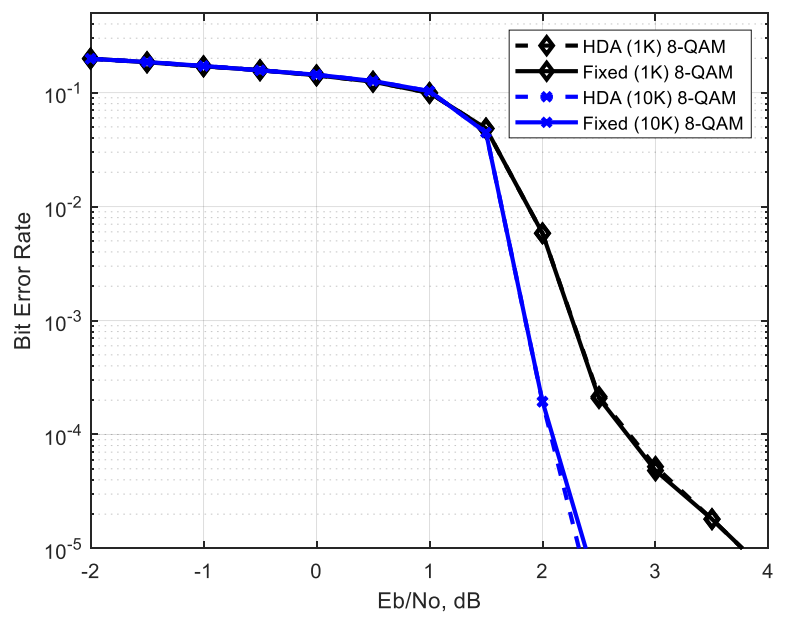

Fig. 17. BER performance for 8-QAM turbo codes with HDA and fixed stopping criteria.

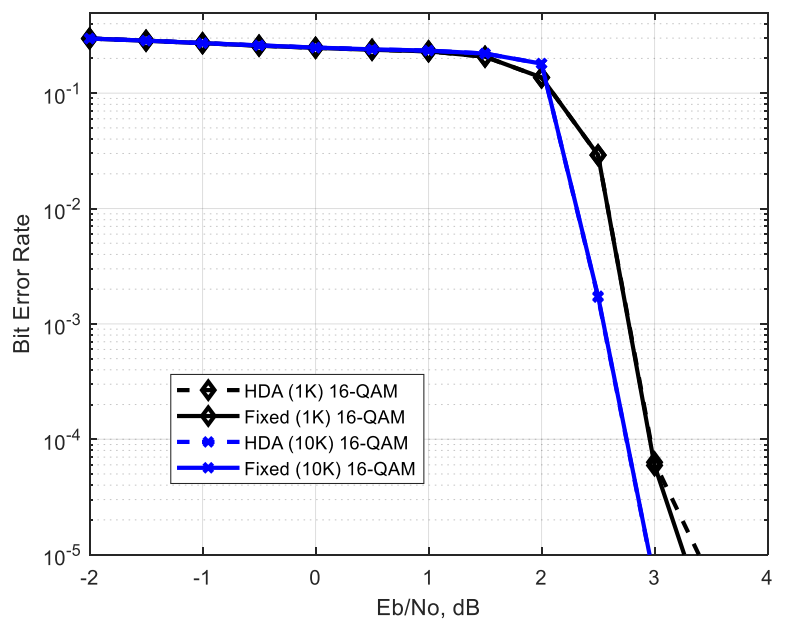

Fig. 18. BER performance for 16-QAM turbo codes with HDA and fixed stopping criteria.

\section{Overall Performance of Iterative Turbo Decoding Stopping Criteria with QAM}

From the results in Sections III $\mathrm{A}$ to $\mathrm{C}$, the BER performances of 4-QAM, 8 QAM and 16 QAM for $1 \mathrm{~K}$ and $10 \mathrm{~K}$ frame sizes for $\mathrm{CE}$ and HDA stopping criteria are maintained successfully. Both stopping criteria only face a very small degradation which is less than $0.2 \mathrm{~dB}$ at high $\mathrm{Eb} / \mathrm{No}$ region. This proves that $\mathrm{CE}$ and HDA stopping algorithm and its thresholds are capable to detect the correct convergence output from turbo decoder and make the correct early termination of the iterative turbo decoder.

Meanwhile for SCR, the stopping algorithm and its threshold result an early termination for iterative turbo decoding. However, this early termination causes the BER degradation that approaching to $0.6 \mathrm{~dB}$ at high $\mathrm{Eb} / \mathrm{No}$ region. This indicates that SCR detects the false convergence output and makes the turbo decoder stops early even though the output is not converged yet. This false detection/false alarm cases results the error in received data and increase the BER degradation.

\section{CONCLUSION}

From the results obtained, it can be concluded that $\mathrm{CE}$ is not suitable for use with higher-order modulation at low Eb/No as it cannot terminate early. However, in high EbNo, CE performs with all the modulations and gives a significant reduction in AIN while maintaining the BER performance. Meanwhile, for SCR, this stopping criterion is capable of terminating early at low and high $\mathrm{Eb} / \mathrm{No}$ for all frame sizes. The SCR is also capable of maintaining the BER performance for small frame sizes while it has a small degradation in BER at high $\mathrm{Eb} / \mathrm{No}$ for large frame sizes. As with SCR, HDA is also capable of terminating early at low and high $\mathrm{Eb} / \mathrm{No}$ for all frame sizes. The HDA is also capable of maintaining the BER performance for small and large frame sizes for various $\mathrm{Eb} /$ No.

\section{ACKNOWLEDGMENT}

The authors would like to thank the Ministry of Education (MOE) for supporting this research through FRGS grant number FRGS/1/2017/ICT02/UITM/02/2.

\section{REFERENCES}

C. Berrou, A. Glavieux, and P. Thitimajshima, "Near Shannon limit error-correcting coding and decoding: Turbo-codes. 1," in Proceedings of ICC '93 - IEEE International Conference on Communications, 1993, vol. 2, pp. 1064-1070 vol.2.

C. E. Shannon, "Probability of error for optimal codes in a Gaussian channel," Bell Syst. Tech. J., vol. 38, no. 3, pp. 611-626, 1929.

C. C. Wang, "On the performance of turbo codes," in IEEE Military Communications Conference. Proceedings. MILCOM 98 (Cat. No.98CH36201), 1998, vol. 3, pp. 987-992 vol.3.

R. Y. Shao, S. Lin, and M. P. C. Fossorier, "Two simple stopping criteria for turbo decoding," IEEE Trans. Commun., vol. 47, no. 8, pp. 1117-1120, 1999.

P. S. Bhanubhai, M. G. Shajan, and U. D. Dalal, "Performance of Turbo Encoder and Turbo Decoder for LTE," Int. J. Eng. Innov. Technol., vol. 2, no. 6, pp. 122-128, 2012.

R. Mohamad and H. Harun, "Enhancement of cross-entropy based stopping criteria via turning point indicator," in 2017 7th 
International Conference on Modeling, Simulation, and Applied Optimization (ICMSAO), 2017, pp. 1-2.

[7] R. Mohamad, H. Harun, M. Mokhtar, W. A. W. Adnan, and K. Dimyati, "Threshold-Based Bit Error Rate for Stopping Iterative Turbo Decoding in a Varying SNR Environment," Frequenz, vol. 71, no. 1-2, pp. 83-94, 2017.

[8] Y. Beeharry, T. P. Fowdur, and K. M. S. Soyjaudah, "Performance of Bit-Level Decoding Algorithms for Binary LTE Turbo Codes with Early Stopping," Istanbul Univ. Electr. Electron. Eng., vol. 17, no. 2, pp. 3399-3416, 2017.

[9] M. AlMahamdy and J. Dill, "Half-iteration early termination of turbo decoding," in Computing and Communication Workshop and Conference (CCWC), 2017 IEEE 7th Annual, 2017, pp. 1-2.

[10] M. S. Aziz, H. Abdel-Kader, and K. Y. Youssef, "Implementation of a smart and power efficient Turbo Decoder using SDR algorithm," in Computer Engineering \& Systems (ICCES), 2011 International Conference on, 2011, pp. 173-177.

[11] L. H. Abderrahmane, "Design of a new interleaver using cross entropy method for turbo coding," IET Commun., vol. 7, no. 9, pp. 828-832, 2013.

[12] Y. Wei, Y. Yang, L. Wei, and W. Chen, "Comments on 'A New Parity-Check Stopping Criterion for Turbo Decoding,"” IEEE Commun. Lett., vol. 16, no. 10, pp. 1664-1667, 2012.

[13] Y. Saouter, "Improving turbocode performance by cross-entropy," in 2011 Wireless Telecommunications Symposium (WTS), 2011, pp. $1-4$. 